\title{
A low-complexity global optimization algorithm for temperature and pollution control in flames with complex chemistry
}

\author{
L. Debiane *, B. Ivorra*, B. Mohammadi*, F. Nicoud* \\ Th. Poinsot ${ }^{\dagger}$, A. Ern ${ }^{\ddagger}$, H. Pitsch ${ }^{\ddagger}$ \\ * University of Montpellier, France \\ $\dagger$ Cerfacs, Toulouse, France \\ $\ddagger$ ENPC, France
}

December 12, 2006

\begin{abstract}
Controlling flame shapes and emissions is a major objective for all combustion engineers. Considering the complexity of reacting flows, new optimization methods are required: this paper explores the application of control theory for partial differential equations to combustion. Both flame temperature and pollutant levels are optimized in a laminar Bunsen burner computed with complex chemistry using a recursive semi-deterministic global optimization algorithm. In order to keep computational time low, the optimization procedure is coupled with mesh adaptation and incomplete gradient techniques.
\end{abstract}

Keyword - Combustion, Bunsen flame, Pollution, Optimization, Incomplete sensitivity, Mesh adaptation.

\section{Introduction}

Control of temperature and species in flames is an important challenge for industrial and environmental issues. Many studies exist on numerical simulation of pollutant formation in flames $[1,2,3,4,5]$. This paper aims to apply control theory for PDEs [6] to flames.

We focus on a simple laminar Bunsen flame simulated with detailed chemistry [7]. We concentrate on the reduction of the Zeldovich-NO, also called 
thermal NO. This is one of the major NO source in a Bunsen flame. We also study the control of temperature distribution in flames which is of importance in combustion engine design. Finally, we pay attention to the control of flame length at a given fuel rate in the flow. These formulations are alternative approaches to consider pollutant control in flames [5] and also have natural applications in the design of combustion chambers.

As the considered functionals are not necessarily convex, we use a recursive semi-deterministic global optimization algorithm. This algorithm allows to escape from local minima at a lower cost than genetic algorithms $[12,13,14]$.

To keep computational time low we test here if it's possible to use approximate state and sensitivity evaluations [8] during optimization. In that sense, different discretizations are used for the computation of the state and gradient and an unstructured mesh adaptation strategy is applied to adapt the mesh to the solution during optimization process $[9,10,11]$. Once the optimization is achieved, the final design is a posteriori validated by an accurate calculation.

Section 2 presents the physical Bunsen flame modeling and the equations to be minimized. In section 3 and 4 , we give a short introduction to the optimization and incomplete gradient methods used in this paper. Finally results are presented and interpreted in section 5 .

\section{Bunsen flame model}

We introduce three non dimensional numbers $\chi_{H_{2}}, \chi_{N_{2}}$ and $\chi_{O_{2}}$, corresponding to the molar fractions in the premixed mixture of species $\mathrm{H}_{2}, \mathrm{~N}_{2}$ and $\mathrm{O}_{2}$, linked by $\chi_{\mathrm{H}_{2}}+\chi_{\mathrm{O}_{2}}+\chi_{N_{2}}=1$.

The Bunsen flame corresponds to the following oxidation reactions (see [1] for the details of the governing equations):

$$
2 \mathrm{H}_{2}+\mathrm{O}_{2} \rightarrow 2 \mathrm{H}_{2} \mathrm{O}
$$

The hydrogen mechanism can be described using nineteen elementary reactions and nine species $\mathrm{H}_{2}, \mathrm{O}_{2}, \mathrm{H}, \mathrm{O}, \mathrm{OH}, \mathrm{HO}_{2}, \mathrm{H}_{2} \mathrm{O}, \mathrm{H}_{2} \mathrm{O}_{2}, \mathrm{~N}_{2}$. The NO specie is added to the mechanism in order to simulate the formation of the Zeldovich-NO.

The domain of study $\Omega$ is presented in Figure 1. We would like to reduce the NO flux of the flame through the section $\Gamma$ defined at $z=1.5 \mathrm{~cm}$.

\subsection{Parameters}

Optimization control parameters are (see Figure 2): 
- The inflow velocity of the premixed mixture $v_{1}$ taken as

$$
v_{1}\left(\overline{v_{1}}, p_{1}, p_{2}\right)=\overline{v_{1}}\left(1-\exp \left(\frac{-\left|r_{0}-r\right|}{r_{1}}\right)\right)+S\left(p_{1}, p_{2}\right)
$$

with $r_{0}=0.2, r_{1}=0.05$ and $S\left(p_{1}, p_{2}\right)=p_{1} r^{1.2} \sin \left(p_{2} r\right) \sin \left(r-r_{0}\right) . p_{1}$ and $p_{2}$ are two free parameters designed to control the flow profile (see below for the definition of the control space).

- The co-flow velocity $v_{2}$ of the form

$$
v_{2}\left(\overline{v_{2}}\right)=\overline{v_{2}}\left(1-\exp \left(\frac{-\left|r_{0}+r_{3}-r\right|}{r_{1}}\right)\right) \text { with } r_{3}=0.05
$$

- The equivalence ratio: $\phi=\frac{\chi_{H_{2}}}{2 \chi_{O_{2}}}$.

- The dilution factor: $\alpha=\frac{\chi_{N_{2}}}{\chi_{O_{2}}}$.

Here $r$ denotes the radial coordinate.

The control space $\Theta$ is included in $\mathbb{R}^{6}$. The vector of control parameters is $x=\left(\overline{v_{1}}, p_{1}, p_{2}, v_{2}, \phi, \alpha\right) \in \Theta=[50,200] \times[-120,120] \times[-50,50] \times[50,200] \times$ $[4,10] \times[0,10]$. It should be noted that a constraint on the fuel rate in the flow implies a compatibility relation between $p_{1}, p_{2}$ and $\overline{v_{1}}$.

\subsection{Cost functions}

In order to reduce the NO flux through $\Gamma$ and at the same time achieve a target temperature profile, we consider the following cost function:

$$
J_{1}(x)=\gamma_{1} \int_{\Gamma} \rho y_{N O} v \cdot n+\gamma_{2} \int_{\Gamma}\left(T-T_{\text {target }}\right)^{2}
$$

where $\rho$ is the density, $y_{N O}$ the mass fraction, $v$ the velocity, $T$ the temperature and $T_{\text {target }}$ a target temperature profile. $\gamma_{1}, \gamma_{2}$ are given positive scalars and $n$ the normal unit vector to $\Gamma$.

Our second objective is to reduce the flame length at a given flow rate using the following cost function:

$$
J_{2}(x)=\int_{r=0} y_{H_{2}} \quad \text { with } \quad \gamma_{1} \int_{z=0} \rho y_{H_{2}} v \cdot n=\text { const. }
$$

$y_{N O}, y_{H_{2}}$ and $T$ are given by the solution of the axisymmetric Bunsen laminar flame code described in [7]. This solver uses an extension to chemically reacting flows of a finite element method. The method uses streamline 
diffusion, least squares stabilization of the pressure gradient, a low-Mach continuity equation and a shock capturing term designed to control species mass fraction under-shoots near flame fronts.

Numerical experiences show that functions $J_{1}$ and $J_{2}$ are non convex [11]. We would like therefore to use a low complexity global minimization algorithm previously applied with success to various nonlinear industrial optimization problems $[13,14,16]$.

\section{Semi-deterministic recursive optimization method}

Many deterministic minimization algorithms, which perform the minimization of a function $J: \Theta \rightarrow \mathbb{R}$, can be seen as discretizations of the following dynamical system $[15,16]$ where $x$ denotes the vector of control parameters belonging to an admissible space $\Theta . x_{0} \in \Theta$ is the initial condition. $\zeta$ is a fictitious parameter. $x_{\zeta}(\zeta)=\frac{d x(\zeta)}{d \zeta}$. $M$ is a local metric transformation and $d$ a direction in $\Theta$.

$$
\left\{\begin{array}{l}
M(\zeta) x_{\zeta}=-d(x(\zeta)) \\
x(\zeta=0)=x_{0}
\end{array}\right.
$$

For example if $d=\nabla J$, the gradient of $J$, and $M=I d$, the identity operator, we recover the classical steepest descent method while with $d=\nabla J$ and $M=\nabla^{2} J$ the Hessian of $J$, we recover the Newton method [17].

We made the following assumptions $[16]: J \in C^{1}(\Theta, \mathbb{R})$ and coercive. The minimum of $J$ is denoted by $J_{m}$. In cases where $J_{m}$ is unknown, we set $J_{m}$ to a lower value (for example $J_{m}=0$ for a non-negative function $J$ ) and look for the best solution for a given complexity and computational effort. This is the approach adopted here where we set $J_{m}=0$ and we predefine the effort we would like to make in the optimization algorithm.

Global optimization with system (3) is valid if the following system has a solution:

$$
\left\{\begin{array}{l}
M(\zeta) x_{\zeta}(\zeta)=-d(x(\zeta)) \\
x(0)=x_{0} \\
J\left(x\left(Z_{x_{0}}\right)\right)=J_{m} \quad \text { with finite } Z_{x_{0}} \in \mathbb{R}
\end{array}\right.
$$

This boundary value problem is over-determined (i.e. two conditions and only one derivative). The previous over-determination is an explanation of why we should not solve global optimization problems with methods which are particular discretizations of initial value problem for first order differential 
equations. We could use variants of classical methods after adding a second order derivative [15]:

$$
\left\{\begin{array}{l}
\eta x_{\zeta \zeta}(\zeta)+M(\zeta) x_{\zeta}(\zeta)=-d(x(\zeta)) \\
x(0)=x_{0}, \quad \dot{x}(0)=\dot{x}_{0} \\
J\left(x\left(Z_{x_{0}}\right)\right)=J_{m}
\end{array}\right.
$$

where $x_{\zeta \zeta}(\zeta)=\frac{d^{2} x(\zeta)}{d \zeta^{2}}$

The over determination can be removed, for instance, by considering $x_{0}=$ $v$ for (3) (resp. $\dot{x}(0)=v$ for (5)) as a new variable to be found by the minimization of $h(v)=J\left(x_{v}\left(Z_{v}\right)\right)-J_{m}$, where $x_{v}\left(Z_{v}\right)$ is the solution of (3) (resp. (5)) found at $\zeta=Z_{v}$ starting from $v$.

The algorithm $A_{1}\left(v_{1}, v_{2}\right)$ reads:

- $\left(v_{1}, v_{2}\right) \in \Theta \times \Theta$ given

- Find $v \in \operatorname{argmin}_{w \in \mathcal{O}\left(v_{2}\right)} h(w)$ where $\mathcal{O}\left(v_{2}\right)=\left\{t \overrightarrow{v_{1} v_{2}}, t \in \mathbb{R}\right\} \cap \Theta$

- return $v$

The line search minimization might fail. For instance, a secant method degenerates on plateau and critical points. In this case, we add an external level to the algorithm $A_{1}$, keeping $v_{1}$ unchanged, and looking for $v_{2}$ by minimizing a new functional $w \rightarrow h\left(A_{1}\left(v_{1}, w\right)\right)$.

This leads to the following two-level algorithm $A_{2}\left(v_{1}, v_{2}\right)$ :

- $\left(v_{1}, v_{2}\right) \in \Theta \times \Theta$ given

- Find $v^{\prime} \in \operatorname{argmin}_{w \in \mathcal{O}\left(v_{2}\right)} h\left(A_{1}\left(v_{1}, w\right)\right)$ where $\mathcal{O}\left(v_{2}\right)=\left\{t \overrightarrow{v_{1} v_{2}}, t \in \mathbb{R}\right\} \cap \Theta$

- return $v^{\prime}$

The choice of initial conditions in this algorithm contains the non-deterministic feature of the algorithm. The construction can be pursued building recursively $h^{i}\left(v_{2}^{i}\right)=\min _{v_{2}^{i} \in \Theta} h^{i-1}\left(A_{i-1}\left(v_{1}, v_{2}^{i}\right)\right)$, with $h^{1}(v)=h(v)$ where $i$ denotes the external level. Mathematical background for this approach and validation on academic test cases and solution of nonlinear PDEs are available $[16,13,14]$.

In practice, this algorithm succeeds if the trajectory passes close enough to the infimum (i.e. in $B_{\varepsilon}\left(x_{m}\right)$ where $\varepsilon$ defines the accuracy in the capture of the infimum). Hence, in the algorithm above $x_{w}\left(Z_{w}\right)$ is replaced by the best solution found over $\left[0, Z_{w}\right]$. 


\section{Multi-level sensitivity analysis}

Combustion computations are CPU consuming. We gather here some ingredients used to reduce the computational complexity of the optimization problem.

We would like to use incomplete state calculation for sensitivity evaluations together with one accurate state evaluation at each design step. This means that during optimization we mostly achieve suboptimal sensitivity analysis and linesearch [8]. This is possible because the semi-deterministic algorithm above requires less accuracy in the definition of sensitivities [13].

Another ingredient is the use of multi-level nonconformal unstructured meshes with interpolation operators between the different levels [9] for sensitivity analysis. Hence, gradient computations are made on rather coarse meshes.

It is impossible to use an uniform fine mesh over the domain to avoid this difficulty as the cost of the calculation will make the optimization impossible to achieve. The minimal mesh size needed will produce a uniform fine mesh of $10^{6}$ nodes, while the meshes here have between 1000 and 5000 nodes for the different level of refinements (see Figure 7).

Therefore, to monitor the accuracy of state calculations and the functional, at each iteration of optimization a full unstructured solution-based mesh adaptation is performed $[9,10,11]$ to keep the mesh and solution compatible (see section 4.1 for a brief description).

Experience shows that if the flame leaves the area of refined mesh the flow calculation, and consequently the optimization, will fail.

Using these ingredients, the cost for optimization is only 30 percent of the overall flame calculations.

\subsection{Mesh adaptation}

This paragraph is devoted to a short description of the mesh adaptation by metric control we use with Delaunay meshes.

Given a positive definite matrix $M(x)$ we define a variable metric by $\|x-y\|^{2}=(x-y)^{T} M(x)(x-y)$. M-circles, i.e. circles with respect to the variable metric, are ellipses in the Euclidean space. A given triangulation is said to satisfy the M-Delaunay criteria if for all inner edges the quadrangle made by the its two adjacent triangles are such that the fourth vertex is outside the M-circle passing by the 3 other vertices.

It can be shown that the Delaunay mesh is the nearest to a quasiequilateral mesh, in the sense that the smallest angle in the triangulation 
is maximized. So, if the local metric is Euclidian, the mesh elements are isotropic. But, anisotropy is introduced by way of the local metric [9].

The definition of the metric is based on the Hessian of the state variables of the problem. Indeed, for a $P^{1}$ Lagrange discretization of a variable $u$, the interpolation error is bounded by:

$$
\mathcal{E}=\left|u-\Pi_{h} u\right|_{0} \leq c h^{2}\left|D^{2} u\right|_{0}
$$

where $h$ is the element size, $\Pi_{h} u$ the $P^{1}$ interpolation of $u$ and $D^{2} u$ its Hessian matrix. This matrix is symmetric and one generates a Riemanian metric making this matrix positive definite with eigenvalues bounded by below and above (this will define the largest and smallest edge in the mesh) [19].

Now, if we generate, by a Delaunay procedure, an equilateral mesh with edges of length of 1 in the metric $\mathcal{M} /(c \mathcal{E})$, the interpolation error $\mathcal{E}$ is equidistributed over the edges of length $a_{i}$ if

$$
\frac{1}{c \mathcal{E}} a_{i}^{T} M a_{i}=1
$$

\section{$5 \quad$ Numerical results}

The optimizations presented here have been performed with the two levels algorithm $A_{2}$ presented above. The effect of each level can be seen in Figure 3: a first plateau is reached and the algorithm allows to reach a second plateau with a global reduction of $90 \%$ of the functional.

Optimization results for functional $J_{1}$ are presented in Figure 4. We can see that the target temperature is almost achieved and that the NO flux is drastically reduced. Initial and final temperature and NO mass fraction distributions are shown in Figure 5.

Optimization results for functional $J_{2}$ are shown in Figure 6. We present the maximum and minimum flame lengths which can be obtained with the current parameterization. There is a difference of $30 \%$ in flame length between both configurations. The injection profiles obtained by maximizing and minimizing the flame length at a given flow rate are quite counter-intuitive (see Figure 8) as the maximum injection along the symmetry line leads to a minimum flame length.

The explanation for this result is twofold:

a) The dihydrogen species has a small characteristic Schmidt number of order 0.2 , indicating fast diffusion/mixing with the ambient air [18]. Injecting the fuel near the centerline produces fast combustion in this region and quick decrease of the H2 mass fraction, 
b) Peripheric injection of fuel generates larger values of the minimized flame length which is based on the centerline mass fraction. Indeed, the H2 species is convected before it diffuses towards the centerline of the jet which is reached further downstream.

Further studies would be required to clarify the effect of the flame length (2) definition on the optimized injection profile. However, this optimization clearly shows the correlation between flame length and pollutant production.

\section{Conclusions}

A new global optimization algorithm based on a recursive search of initial conditions of classical optimization methods has been applied to the control of pollution, temperature and flame length in a Bunsen flame simulated with complex chemistry. This optimization technique combined with an incomplete gradient technique allows to perform low-cost global optimization. It has been shown that controlling the fuel rate in the flow and the main and co-flow inflow velocity profiles is enough to achieve the targeted temperature, NO flux and flame shapes. The approach is currently used in order to improve the design of combustion chambers at Cerfacs for Snecma and Turbomeca companies accounting for environmental issues.

Acknowledgements The authors would like to thank Professors P. Moin, D. Cabale, M. Chethik and D. Micheal for their deep interest and helpful comments and orientations during this work realization.

\section{References}

[1] Williams, F.A. Combustion Theory. 1985. Addison-Welsey

[2] Pitsch, H. Improved Pollutant Predictions in Large-Eddy Simulation of Turbulent Non-Premixed Combustion by Considering Scalar Dissipation Rate Fluctuations. Proc. Comb. Inst., 29, 1971:1978. 2002

[3] Warnatz, J. Concentration, Pressure and Temperature dependence of the flame velocity in Hydrogen-Oxygen-Nitrogen Mixtures. Combustion Science and Technologie. 26, 203:213. 1981

[4] Pitsch, H. \& Barths, H. \& Peters, N. Three-Dimensional Modeling of NOx and Soot Formation in DI-Diesel Engines Using Detailed Chemistry Based on the Interactive Flamelet Approach. SAE 962057. 1996 
[5] Peters, N. \& Donnerhack, S. Structure and similarity of nitric oxide production in turbulent diffusion flames. 18th International Symposium on Combustion. The Combustion Institute, 33:42. 1981

[6] Lions, J.L. Oeuvres choisies de Jacques-Louis Lions. Vol. II : Contrôle - Homogénéisation. 2003. EDP Science.

[7] Burman, E. \& ERn, A. \& V. Giovangigli Bunsen flame simulation by finite elements on adaptively refined, unstructured triangulations, Combustion Theory Modelling 8 (1), 65:84. 2004

[8] Mohammadi, B. \& Pironneau, O. Applied shape optimization for fluids, 2001. Oxford Univ. Press.

[9] Frey, P.J. \& George, P.L.Mesh generation: application to finite elements. 2001. Paris: Hermès Sci. Publications.

[10] FRey, P.J. Yams: A fully automatic adaptive isotropic surface remeshing procedure. INRIA RT-0252. 2001

[11] Debiane, L. Application de l'adaptation de maillages en mecanique des fluides, combustion et traitement de l'image. 2004. Ph.D. University of Montpellier.

[12] Goldberg, D. Genetic algorithms in search, optimization and machine learning. 1989. Addison Wesley.

[13] Ivorra, B. \& Mohammadi, B. \& Santiago, J.G.\& Hertzog, D.E. Semi-deterministic and genetic algorithms for global optimization of microfluidic protein folding devices. International Journal of Numerical Methods in Engineering. To be published. 2005

[14] Ivorra, B. \& Mohammadi, B. \& Redont, P.\& Dumas, L. Semideterministic vs genetic algorithms for global optimization of multichannel Optical Filters. International Journal of Computational Science and Engineering. To be published. 2006

[15] Atrouch, H. \& Cominetti, R. A dynamical approach to convex minimization coupling approximation with the steepest descent method,J. Differential Equations, 128 (2), 519-540. 1996

[16] Mohammadi, B. \& Saiac, J. H. Pratique de la simulation numérique, 2002. Dunod, Paris. 
[17] VAnderplaAts, G.N. Numerical optimization techniques for engineering design. 1990. Mc Graw-Hill.

[18] Giovangigli, V. Multicomponent flow modeling. 1999. Birkhauser

[19] Hecht, F. Mohammadi, B. Mesh Adaptation by Metric Control for Multi-scale Phenomena and Turbulence, AIAA 97-0859. 1997. 


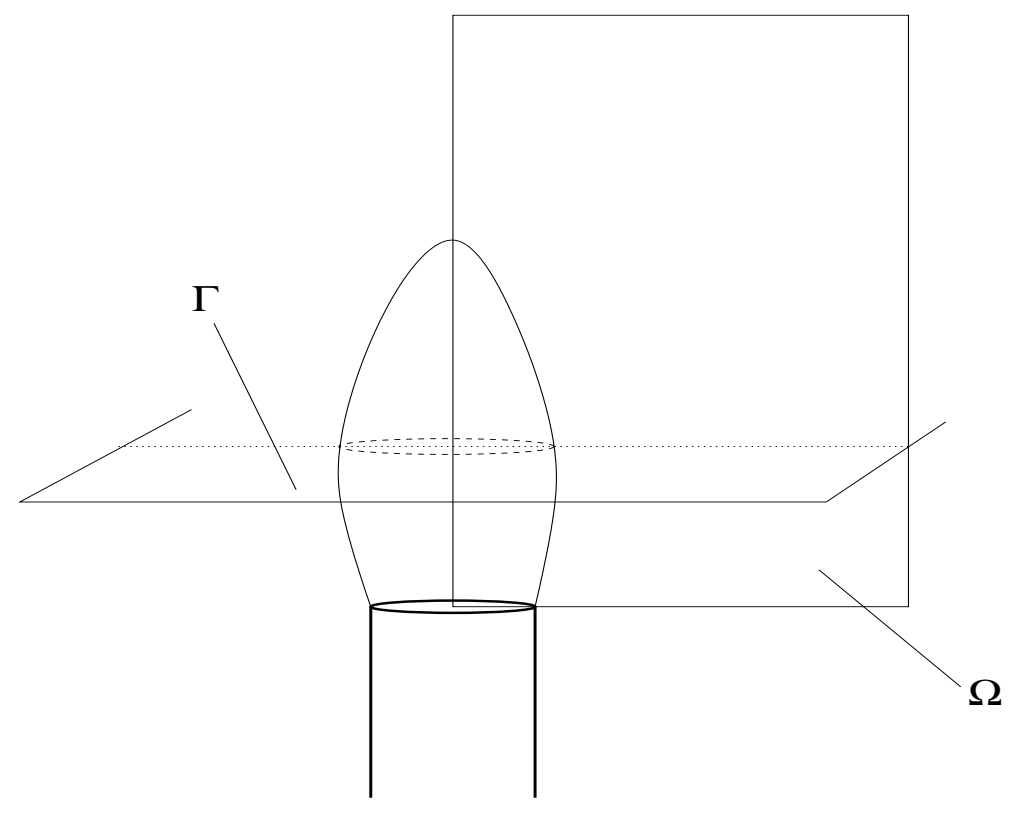

Figure 1: Bunsen flame: the NO flux is evaluated through $\Gamma=\{z=1.5 \mathrm{~cm}\}$ into the computational domain $\Omega$.

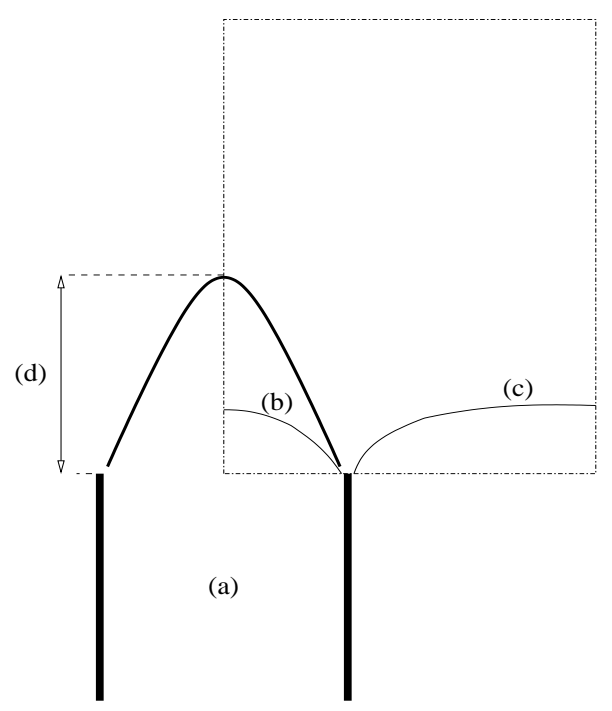

Figure 2: Illustration of the parameterizations: $(a)$ is the premixed mixture $\mathrm{H}_{2}, \mathrm{O}_{2}, \mathrm{~N}_{2}$ injected with velocity $v_{1}$ shown on $(b)$, the co-flow velocity $v_{2}$ $(c)$ and the flame length $(d)$. 


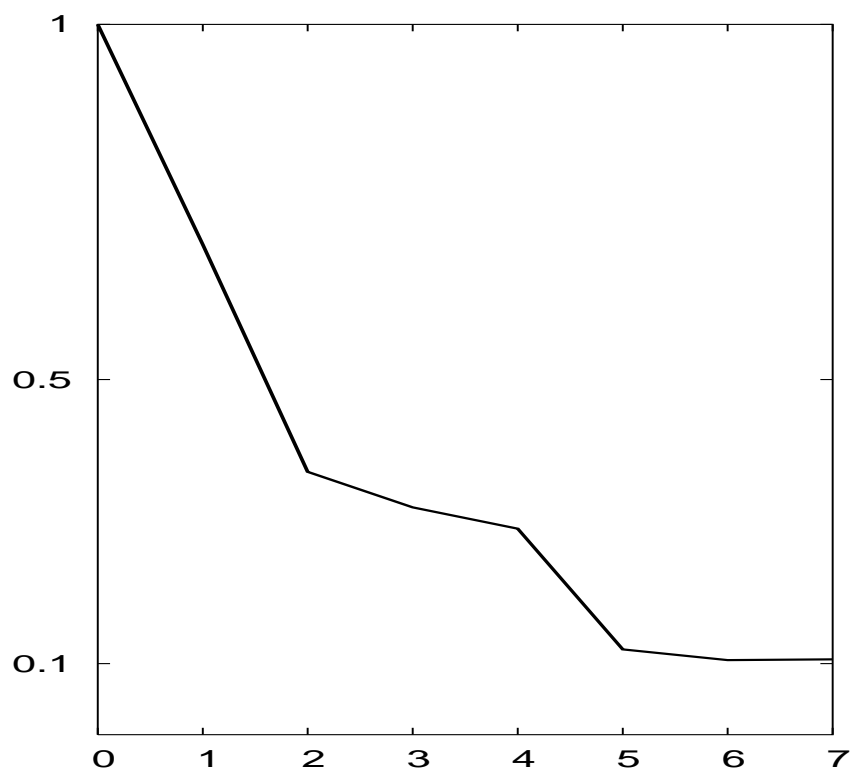

Figure 3: NO reduction: Functional $J_{1}$ evolution vs. accumulation of optimization iterations. The two-level algorithm allows to escape from local minima. Each iteration reports the best element found by the core minimization algorithm (the inner level).
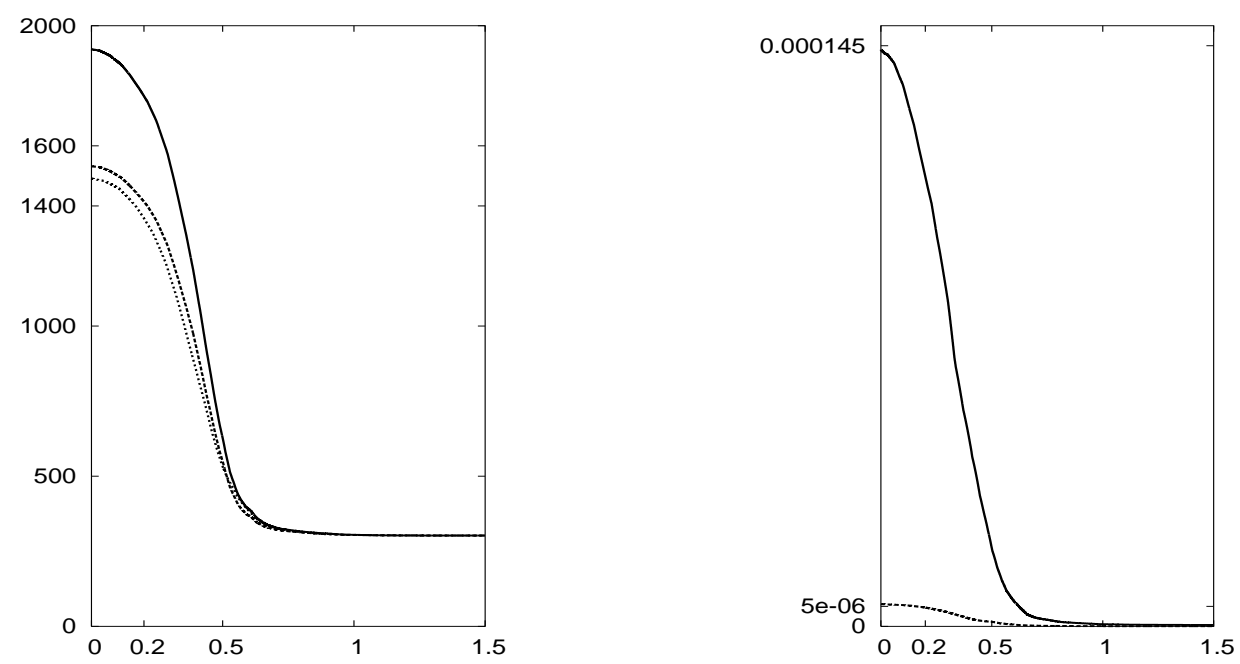

Figure 4: Left: initial (top), target (center) and optimized (bottom) temperature profiles $(\mathrm{K})$ along $z=1.5 \mathrm{~cm}$ (x-axis in $\mathrm{cm}$ ). Right: NO flux $\left(\mathrm{gcm}^{-1} \mathrm{~s}^{-2}\right)$ through $z=1.5 \mathrm{~cm}$ before (top) and after (bottom) optimization. 

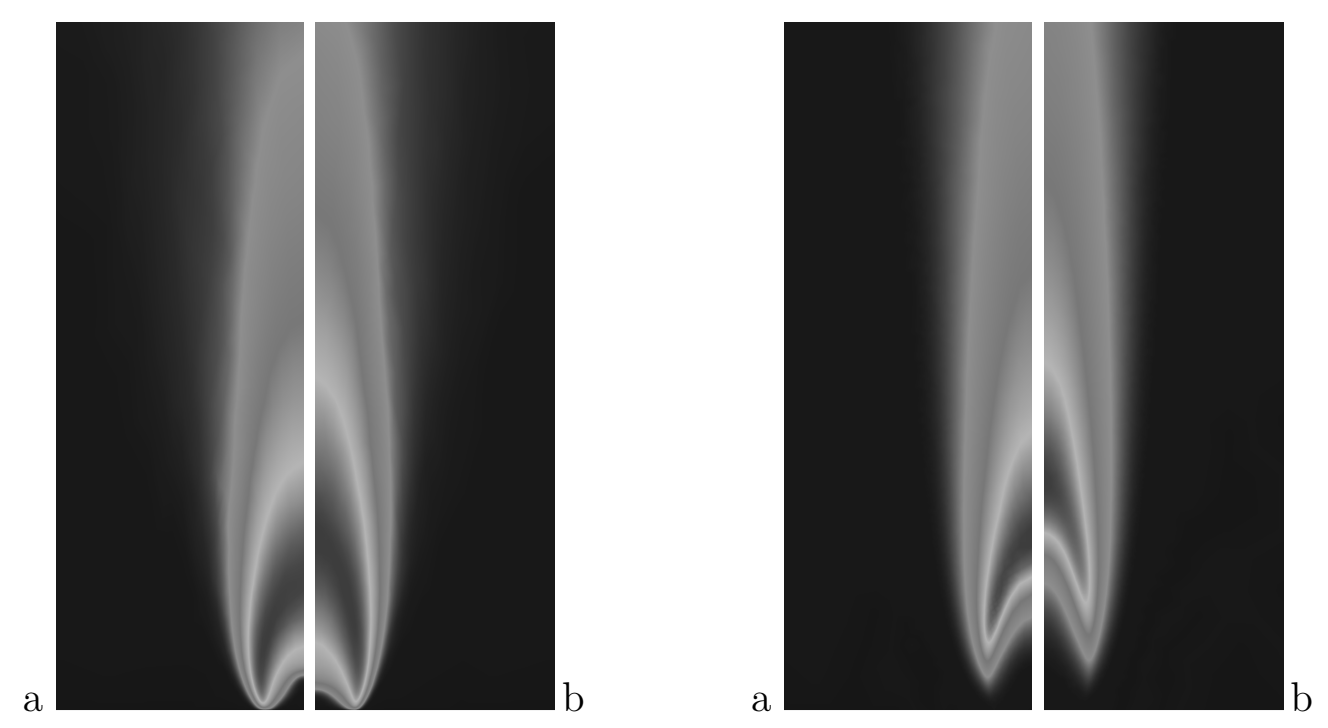

Figure 5: Optimized $(a)$ and initial $(b)$ temperature (left) and NO mass fraction (right) distributions.
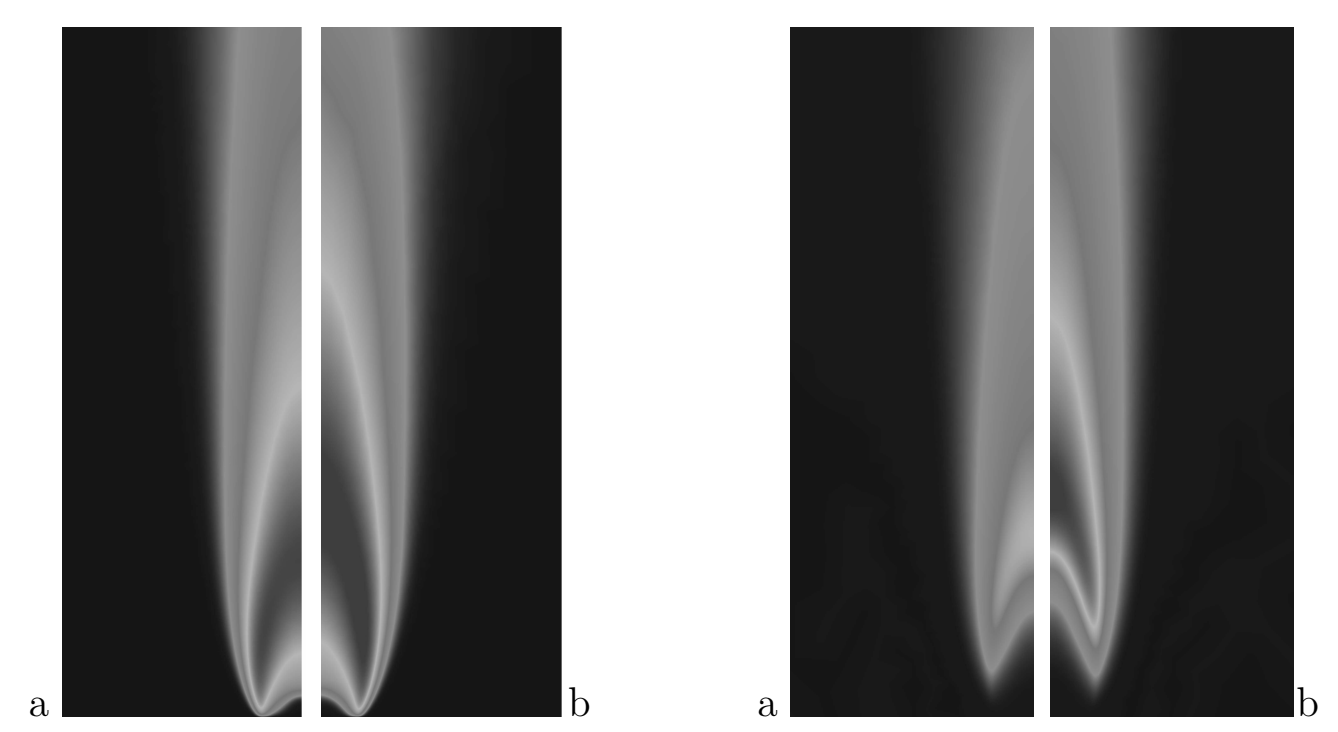

Figure 6: Temperature contours (left) and NO mass fraction contours (right) for minimal $(a)$ and maximal $(b)$ flame length. There is a clear correlation between flame length and NO production. 


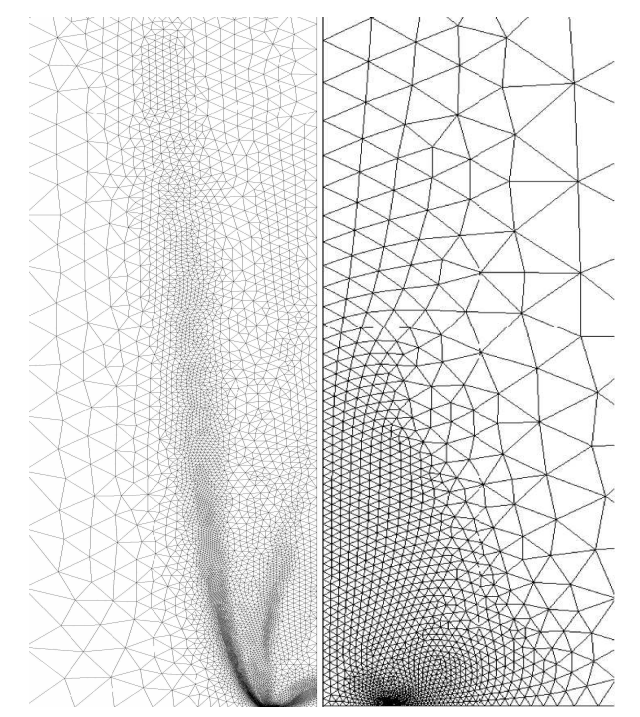

Figure 7: A typical solution-based adapted mesh (left) and the coarse level mesh used for sensitivity evaluation (right).

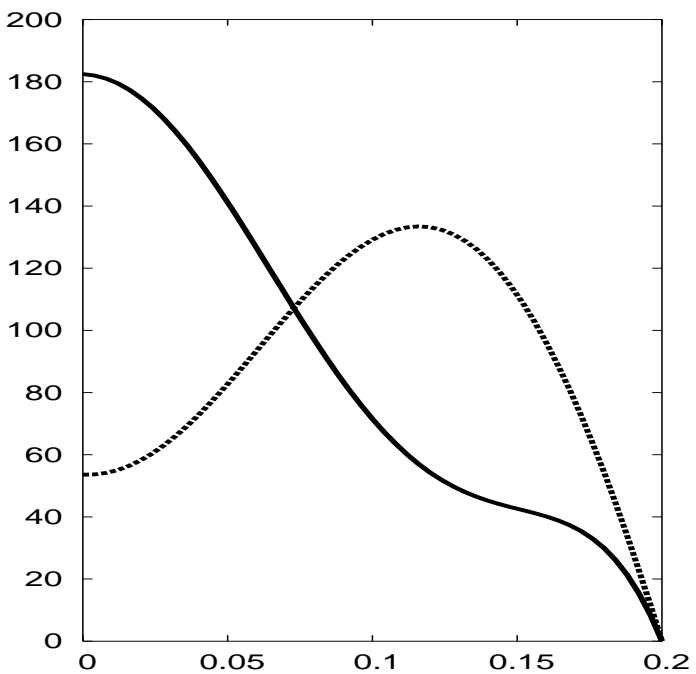

Figure 8: The injection velocity $\left(\mathrm{cms}^{-1}\right)$ profiles are quite counter-intuitive (x-axis in $\mathrm{cm}$ ). The continuous profile minimizes the flame length of and the dash profile maximizes it. 